\title{
RELATIVITY PRINCIPLES IN $1+1$ DIMENSIONS AND DIFFERENTIAL AGING REVERSAL
}

\author{
E. Minguzzi \\ Department of Applied Mathematics, Florence University, \\ Via S. Marta 3, I-50139 Florence, Italy \\ and INFN, Piazza dei Caprettari 70, I-00186 Roma, Italy \\ ettore.minguzzi@unifi.it
}

\begin{abstract}
We study the behavior of clocks in $1+1$ spacetime assuming the relativity principle, the principle of constancy of the speed of light and the clock hypothesis. These requirements are satisfied by a class of Finslerian theories parametrized by a real coefficient $\beta$, special relativity being recovered for $\beta=0$. The effect of differential aging is studied for the different values of $\beta$. Below the critical values $|\beta|=1 / c$ the differential aging has the usual direction - after a round trip the accelerated observer returns younger than the twin at rest in the inertial frame - while above the critical values the differential aging changes sign. The non-relativistic case is treated by introducing a formal analogy with thermodynamics.
\end{abstract}

Key words: clock problem, Finsler spacetimes, anisotropic spacetimes.

\section{INTRODUCTION}

Since their discovery the Lorentz transformations have been derived in many ways $22,10,13,14,9$. The goal was essentially that of reducing the number of postulates making them logically and physically independent. In particular, some authors recognized the need of an isotropy assumption in the derivation [2, 15, 11, 21]. In $1+1$ dimensions the group of rotations is replaced by the group of space reflections, and hence the isotropy assumption is replaced by a parity assumption.

In this work we consider a 1+1-dimensional theory that does not satisfy the parity symmetry assumption although it is compatible with the usual principles of special relativity. Its existence is due to the presence (in $1+1$ dimensions) of the Lorentz group representation $\Lambda \rightarrow e^{\beta \theta}$ where $\theta$ is the rapidity and $\beta$ is an arbitrary constant. In this way one can consider 
$e^{\beta \theta} \Lambda$ as the coordinate transformation that relates different inertial frames. The ultimate task is to study the behavior of clocks in the corresponding generalized theories showing an interesting phenomenon that may occur varying the value of $\beta$ : the differential aging reversal.

This class of theories, dependent on the parameter $\beta$ were studied by Bogoslovsky [4] who also generalized them to the higher 3+1-dimensional case. He considered a spacetime $M$ endowed with a Finsler metric of the form $\left(\mathbf{e}^{2}=1, \mathbf{e}=\right.$ cnst., $\left.1 / c \leq \beta<0\right)$

$$
c^{2} \mathrm{~d} \tau^{2}=(c \mathrm{~d} t-\boldsymbol{e} \cdot \mathrm{d} \mathbf{x})^{-2 \beta c}\left(c^{2} \mathrm{~d} t^{2}-\mathrm{d} \mathbf{x}^{2}\right)^{1+\beta c}
$$

i.e. with an expression homogeneous of second degree in the differentials. Here $\beta$ is a universal constant with the dimension of the inverse of a velocity. Bogoslovsky used the dimensionless constant $r=-\beta c$, but we prefer to consider $\beta$ as a universal constant since it will allow us to obtain a finite non-relativistic limit. Finsler metrics are a simple generalization of Riemannian metrics (quadratic forms in the differentials) and were considered by Riemann himself in his seminal works on differential geometry. Finsler geometry is now a mathematically mature subject [20].

Physically, Finsler geometry provides a natural tool for the description of anisotropic spacetimes [1]. For instance, the previous metric is not invariant under the full Poincaré group, as only the Lorentz transformations that send the null vector $n^{\mu}=(1, \boldsymbol{e})$, into a vector proportional to itself may leave the metric unchanged [6, 5, 3. It is clear that the invariance under the group of Lorentz transformation is lost because the spatial $S O(3)$ symmetry is broken into a $O(1)$ symmetry about e. Nevertheless, the Einstein addition of velocity rule holds true because the generalized Lorentz transformations are just ordinary Lorentz transformations times a factor [4, 5, 3. The breaking of rotational invariance reduces to a breaking of the parity symmetry in the $1+1$ case. Hence in this particular case the theory is invariant under the entire proper orthochronous Lorentz group.

A first interesting consequence of the metric is the fact that the light cones defined by $\mathrm{d} \tau^{2}=0$ coincide with those of Minkowski spacetime. Thus it does not introduce any change in the usual causal structure. One can still identify the massive particles with the future directed timelike worldlines.

The symbol $\tau$ for the metric line element suggests that it represents the time of a particle along the particle worldline. This interpretation is correct and will be proved below. We shall call that time proper time although it should not be confused with the proper time of special relativity as it has a different dependence on the velocity of the particle.

In this work we consider the clock problem of special relativity where an accelerated observer makes a round-trip with respect to an inertial observer. Their clocks previously synchronized are compared at the end of the trip. The differential aging is the difference between the proper time of the clock at rest in the inertial frame and the proper time of the accelerating clock at the final meeting event. Special relativity predicts that the differential 
aging has always a positive sign independently of the arbitrary motion performed during the round-trip by the non-inertial observer. A similar result will be proved below in a $1+1$ theory for which $\beta \in \mathbb{R}$ and such that when $\beta$ crosses the values $\beta= \pm 1 / c$ the differential aging changes sign.

Notice that while in special relativity the expression $\tau=\int \sqrt{1-v^{2}} \mathrm{~d} t$ with $\sqrt{1-v^{2}} \leq 1$ implies $\tau \leq t$ in a round trip, in the generalized theories for $\beta \neq 0$ the integrand may be above or below 1 depending on time $t$. A priori, it is not clear that the balance of the contributions should be independent of the round trip followed.

Our aim is also that of obtaining an expression for the differential aging in terms of the acceleration history of the non-inertial observer. Indeed, as we shall see, given that relation we will be able to prove the sign of the differential aging using Holder's inequality. An analogous problem was studied in the special relativistic $1+1$-dimensional case $(\beta=0)$ in [18. The idea is that the proper acceleration of the inertial observer can be measured with a comoving accelerometer and hence it is unambiguous and observable. On the contrary expressions like $\tau=\int \sqrt{1-v^{2}} \mathrm{~d} t$ involve the velocity which depends on the convention of distant simultaneity adopted. The differential aging versus acceleration formulas, being independent of conventions, give more insights on the physical spacetime structure. Connected to them is the problem of autonomous spacetime navigation that will not be discussed here.

The problem of obtaining the differential aging from the acceleration may become quite involved in the $3+1$ case. Indeed, it is difficult to obtain the motion from the decomposition of the proper acceleration with respect to a Fermi-Walker transported tetrad. Even the analogous Riemannian problem has not yet been solved, although the uniqueness of the solution of the Frenet-Serret equations is known since a long time. Thus, for computational reasons we shall consider only the 1+1-dimensional case or, which is the same, motions in $3+1$ dimensions contained in a timelike plane.

\section{PROPER TIME IN FINSLER SPACETIMES}

In the $1+1$ spacetime $M$ the Finslerian line element can be conveniently written

$$
\begin{aligned}
c^{2} \mathrm{~d} \tau^{2} & =(c \mathrm{~d} t-\mathrm{d} x)^{-2 \beta c}\left(c^{2} \mathrm{~d} t^{2}-\mathrm{d} x^{2}\right)^{1+\beta c} \\
& =(c \mathrm{~d} t+\mathrm{d} x)^{2 \beta c}\left(c^{2} \mathrm{~d} t^{2}-\mathrm{d} x^{2}\right)^{1-\beta c} \\
& =(c \mathrm{~d} t+\mathrm{d} x)^{1+\beta c}(c \mathrm{~d} t-\mathrm{d} x)^{1-\beta c}
\end{aligned}
$$

If $|\beta|<1 / c$ the causal structure coincides with that of Minkowski spacetime. If $\beta>1 / c$ the chronological structure coincides with that of Minkowski spacetime and null particles of velocity $\mathrm{d} x / \mathrm{d} t=c$ do not exist. If $\beta<-1 / c$ the chronological structure coincides with that of Minkowski spacetime and 
null particles of velocity $\mathrm{d} x / \mathrm{d} t=-c$ do not exist. The case $|\beta|=1 / c$ will be discussed below.

Let $K^{\prime}$ be a inertial frame in uniform motion with respect to $K$ at a velocity $v$. We define the rapidity $\theta$ with

$$
e^{\theta / c}=\sqrt{\frac{1+v / c}{1-v / c}},
$$

or $\tanh \theta / c=v / c$. In our convention $\theta$ has the dimension of a velocity. In this way we obtain the right limit $\theta=v$ if $c \rightarrow \infty$, which is particularly convenient as the relative frame velocity $v$ is an additive parameter in nonrelativistic physics.

The coordinate transformations that leave the metric invariant are

$$
\begin{aligned}
& c t^{\prime}=e^{\beta \theta}[\cosh (\theta / c) c t-\sinh (\theta / c) x]=\left[\frac{1+v / c}{1-v / c}\right]^{\beta c / 2} \frac{c t-\frac{v}{c} x}{\sqrt{1-v^{2} / c^{2}}}, \\
& x^{\prime}=e^{\beta \theta}[-\sinh (\theta / c) c t+\cosh (\theta / c) x]=\left[\frac{1+v / c}{1-v / c}\right]^{\beta c / 2} \frac{x-v t}{\sqrt{1-v^{2} / c^{2}}}
\end{aligned}
$$

with $\theta \in \mathbb{R}$ and form a proper orthocronous Lorentz group. The group structure reveals that all the inertial reference frames are equivalent and the equation $\mathrm{d} \tau^{2}=0 \Rightarrow c^{2} \mathrm{~d} t^{2}-\mathrm{d} x^{2}=0$ implies that the speed of light is $c$ in every frame. Thus the two principles of special relativity hold

- First principle. A frame moving uniformly with respect to an inertial frame is also an inertial frame.

- Second principle. The velocity of light $c$ is invariant under inertial frame changes.

However, since we do not assume invariance under reflection $x \rightarrow-x$, special relativity in $1+1$ dimensions is not recovered.

Now, notice that in its own coordinates, the motion of $K^{\prime}$ reads $x^{\prime}=0$, and from the invariance of the Bogoslovsky metric we obtain $\mathrm{d} t^{\prime}=\mathrm{d} \tau$. Now, consider the arbitrary motion of an observer $O$ along a timelike curve $\gamma$. At any instant there is an inertial frame $K^{\prime}$ which is instantaneously at rest with $O$. We use the locality principle [17, 16] for clocks measurements, sometimes referred to as the clock hypothesis [19]. It states that the infinitesimal time elapsed in an accelerating frame equals the infinitesimal time elapsed in the local inertial frame $K^{\prime}$. We conclude that the natural metric parametrization $\tau$ represents the proper or natural time of the non-inertial observer. This fact justifies the symbol $\tau$ for the line element.

If $|\beta|=1 / c$ then using Eqs. (21)-(31) we find $c \mathrm{~d} \tau=c \mathrm{~d} t+\operatorname{sgn}(\beta) \mathrm{d} x$ which means that in this case there is no differential aging and the transport of clocks leads to the definition of a global time function $t+\operatorname{sgn}(\beta) x / c$. This global simultaneity definition, in which the simultaneity hyperplanes 
coincide with the null hyperplanes orthogonal to $n$, has been studied from different perspectives in [12, 7, 8, 6] and makes sense in higher dimensional spacetimes as well.

\subsection{The non-relativistic limit}

The non-relativistic, $c \rightarrow+\infty$, limit of Eqs. (2)-(3) is

$$
\mathrm{d} \tau^{2}=e^{2 \beta \mathrm{d} x / \mathrm{d} t} \mathrm{~d} t^{2}
$$

which is still a (degenerate) Finsler metric which seems to have not received attention in the literature. We recall that in the same limit $\theta=v$. Thanks to our dimensional choices for the quantities $\beta$ and $\theta$ we have obtained a meaningful non-relativistic limit. The invariance group is made by transformations of the form

$$
\begin{aligned}
t^{\prime} & =e^{\beta v} t, \\
x^{\prime} & =e^{\beta v}(x-v t),
\end{aligned}
$$

and can be regarded as the $c \rightarrow+\infty$ of their relativistic counterparts. The classical Galilei Physics is obtained if $\beta=0$ in which case $\mathrm{d} \tau=\mathrm{d} t$ and again there is a global absolute time $t$ measured by any (moving) clock. However, if $\beta \neq 0$ the natural time depends indeed on the path $x(t)$ followed, although Eq. (9) clearly shows that the concept of simultaneity is

independent of the inertial frame chosen (i.e. we have absolute simultaneity but not absolute time).

\section{THE TIME DILATION-ACCELERATION FOR- MULA}

We have obtained that the proper time $\tau$ of the non-inertial observer is given by

$$
\tau=\int_{\gamma}(1+v / c)^{(1+\beta c) / 2}(1-v / c)^{(1-\beta c) / 2} \mathrm{~d} t
$$

if $c<+\infty$ and by

$$
\tau=\int_{\gamma} e^{\beta v} \mathrm{~d} t
$$

if $c \rightarrow+\infty$. Special relativity corresponds to the case $(\beta=0, c<+\infty)$, classical Galilei physics to the case $(\beta=0, c \rightarrow+\infty)$. The equation (12) can be obtained from the relativistic limit of (11).

Equations (11)-(12) are not invariant under reflections $x(t) \rightarrow-x(t)$. Thus a clock behaves differently depending on the direction of motion, at least if we are not in the special relativistic or classical case where $\beta=0$. 


\begin{tabular}{|c|c|c|c|}
\hline & $\begin{array}{c}\text { Einsteinian relativity } \\
\qquad 0<c<+\infty \\
\text { Relative simultaneity }\end{array}$ & & $\begin{array}{l}\text { Galilean relativity } \\
\qquad c=+\infty \\
\text { Absolute simultaneity }\end{array}$ \\
\hline$\beta=0$ & $\begin{array}{l}\text { Special relativity, } \\
\text { Proper time, } \\
t(\bar{\tau})>\bar{\tau}\end{array}$ & $\beta=0$ & $\begin{array}{l}\text { Classical physics, } \\
\qquad t(\bar{\tau})=\bar{\tau} \\
\text { Absolute time: } t\end{array}$ \\
\hline $0<|\beta|<1 / c$ & $t(\bar{\tau})>\bar{\tau}$ & \multirow{3}{*}{$\beta \neq 0$} & \multirow{3}{*}{$t(\bar{\tau})<\bar{\tau}$} \\
\hline$|\beta|=1 / c$ & $\begin{array}{c}t(\bar{\tau})=\bar{\tau} \\
\text { Absolute time: } \\
t+\operatorname{sgn}(\beta) x / c\end{array}$ & & \\
\hline $1 / c<|\beta|<+\infty$ & $t(\bar{\tau})<\bar{\tau}$ & & \\
\hline
\end{tabular}

Table 1: The parameters $c$ and $\beta$ determine the physical theory in $1+1$ dimensions. Given a round trip in an inertial frame, $t(\bar{\tau})$ represents the time elapsed for a clock at rest in the inertial frame while $\bar{\tau}$ is the time elapsed in the accelerating frame. The differential aging is $\Delta=t(\bar{\tau})-\bar{\tau}$. At $|\beta|=1 / c$ the differential aging changes sign (differential aging reversal).

If one adds a parity symmetry principle to the relativity principles and the clock hypothesis then the usual special relativity or classical physics is obtained. If this hypothesis is removed, introducing, for instance, a small but non vanishing $\beta$, then alternative theories for the behavior of clocks are obtained.

In this section we consider two clocks. The clock $C_{0}$ is at rest in the origin of an inertial frame $K$. Its proper time is denoted by $t$ and coincides with K's inertial time coordinate. The clock $C_{1}$ of proper time $\tau$ intersects $C_{0}$ 's worldline at two events. For semplicity assume that the two clocks have been synchronized at the first meeting point in such a way that $t=$ $\tau=0$, and let $\bar{\tau}$ be $C_{1}$ 's proper time at the second meeting point. We are going to determine the differential aging $t(\bar{\tau})-\bar{\tau}$ starting from $C_{1}$ 's acceleration $a(\tau)$ during the journey. Table 1 summarizes the results that will be proved in this section.

From the differential relation between $\mathrm{d} t$ and $\mathrm{d} \tau$ we find

$$
\begin{aligned}
t(\tau) & =\int_{0}^{\tau} e^{-\beta \theta\left(\tau^{\prime}\right)} \cosh \left[\theta\left(\tau^{\prime}\right) / c\right] \mathrm{d} \tau^{\prime}, \\
x(\tau) & =\int_{0}^{\tau} e^{-\beta \theta\left(\tau^{\prime}\right)} c \sinh \left[\theta\left(\tau^{\prime}\right) / c\right] \mathrm{d} \tau^{\prime} .
\end{aligned}
$$

Consider an infinitesimal boost in which the rapidity change is $\Delta \theta$. With respect to the local inertial frame $K^{\prime}$ instantaneously at rest with 
$C_{1}, \Delta \theta=\Delta v$ up to higher order terms and thus one recovers $a=\mathrm{d} \theta / \mathrm{d} \tau$ as in ordinary special relativity,

$$
\theta(\tau)=\int_{0}^{\tau} a\left(\tau^{\prime}\right) \mathrm{d} \tau^{\prime}+c \tanh ^{-1}(v(0) / c),
$$

where $v(0)$ is $C_{1}$ 's velocity with respect to $K$ at the first intersection point. At time $\bar{\tau}$, since $C_{1}$ returns in $K$ 's origin, we have $x(\bar{\tau})=0$. Using this relation in the formula for $x(\bar{\tau})$ we find

$$
e^{2 \tanh ^{-1}(v(0) / c)}=\frac{\int_{0}^{\bar{\tau}} e^{-\left(\beta+\frac{1}{c}\right) \int_{0}^{\tau^{\prime}} a\left(\tau^{\prime \prime}\right) \mathrm{d} \tau^{\prime \prime}} \mathrm{d} \tau^{\prime}}{\int_{0}^{\bar{\tau}} e^{-\left(\beta-\frac{1}{c}\right) \int_{0}^{\tau^{\prime}} a\left(\tau^{\prime \prime}\right) \mathrm{d} \tau^{\prime \prime}} \mathrm{d} \tau^{\prime}} .
$$

Plugging this in the equation for $t(\bar{\tau})$ we arrive at the time dilation-acceleration formula

$$
\begin{aligned}
t(\bar{\tau})= & {\left[\int_{0}^{\bar{\tau}} e^{\frac{1}{c}(1-\beta c) \int_{0}^{\tau^{\prime}} a\left(\tau^{\prime \prime}\right) \mathrm{d} \tau^{\prime \prime}} \mathrm{d} \tau^{\prime}\right]^{\frac{1+\beta c}{2}} } \\
& \times\left[\int_{0}^{\bar{\tau}} e^{-\frac{1}{c}(1+\beta c) \int_{0}^{\tau^{\prime}} a\left(\tau^{\prime \prime}\right) \mathrm{d} \tau^{\prime \prime}} \mathrm{d} \tau^{\prime}\right]^{\frac{1-\beta c}{2}},
\end{aligned}
$$

that will be central in what follows. It generalizes a formula given in 18 for the case $\beta=0$. In particular if $v(0)=0$, whatever the choice of sign, we have

$$
t(\bar{\tau})=\int_{0}^{\bar{\tau}} e^{-\left(\beta \pm \frac{1}{c}\right) \int_{0}^{\tau^{\prime}} a\left(\tau^{\prime \prime}\right) \mathrm{d} \tau^{\prime \prime}} \mathrm{d} \tau^{\prime} .
$$

\subsection{The relativistic case}

Let us consider the $c<+\infty$ case. Introduce the function

$$
f(\tau)=e^{\frac{1}{2 c}\left(1-\beta^{2} c^{2}\right) \int_{0}^{\tau} a\left(\tau^{\prime}\right) \mathrm{d} \tau^{\prime}} .
$$

The time dilation-acceleration equation can be rewritten

$$
t(\bar{\tau})=\left[\int_{0}^{\bar{\tau}} f^{\frac{2}{1+\beta c}} \mathrm{~d} \tau^{\prime}\right]^{\frac{1+\beta c}{2}}\left[\int_{0}^{\bar{\tau}}\left(f^{-1}\right)^{\frac{2}{1-\beta c}} \mathrm{~d} \tau^{\prime}\right]^{\frac{1-\beta c}{2}} .
$$

We have to consider different cases,

\subsubsection{The case $|\beta|<1 / c$.}

Consider Eq. (20). From Holder's inequality with $1 / p=\frac{1+\beta c}{2}, 1 / q=\frac{1-\beta c}{2}$, $p, q \in(1,+\infty)$ and $\frac{1}{p}+\frac{1}{q}=1$, it follows $\bar{\tau} \leq t(\bar{\tau})$. If $f$ is not proportional to $f^{-1}$ the inequality is strict, which means that it is strict unless $a(\tau)=0$, that is unless $C_{1}$ and $C_{0}$ follow the same trajectory for $\tau \in[0, \bar{\tau}]$. 


\subsubsection{The case $|\beta|=1 / c$.}

This case has been considered in section 2 where we showed that there is no differential aging and the transport of clocks defines an absolute time $t+\operatorname{sgn}(\beta) x / c$ with null simultaneity slices (lightlike simultaneity).

This is in any case a good simultaneity definition as timelike particles move forward with respect to the absolute time. The absolute time $t+$ $\operatorname{sgn}(\beta) x / c$ is a Lorentz invariant as follows from the general expression of the Lorentz transformation for $\beta \neq 0$,

$$
\begin{aligned}
t^{\prime}+x^{\prime} / c & =\left(\frac{1+v / c}{1-v / c}\right)^{\frac{\beta c-1}{2}}(t+x / c), \\
t^{\prime}-x^{\prime} / c & =\left(\frac{1+v / c}{1-v / c}\right)^{\frac{\beta c+1}{2}}(t-x / c) .
\end{aligned}
$$

3.1.3 The case $|\beta|>1 / c$.

We consider only the case $\beta>1 / c$, the case $\beta<-1 / c$ being analogous. Let $a \in \mathbb{R}$ be such that $\frac{\beta c-1}{2}<a<\frac{1+\beta c}{2}$ and let

$$
\begin{aligned}
p & =\frac{1+\beta c}{2 a} \in(1,+\infty), \\
q & =\frac{2 a}{\beta c-1} \in(1,+\infty) . \\
g(\tau) & =f(\tau)^{1 / a} .
\end{aligned}
$$

We can rewrite Eq. (20) as

$$
\frac{t(\bar{\tau})}{\bar{\tau}}=\left\{\frac{\left(\frac{1}{\bar{\tau}} \int_{0}^{\bar{\tau}} g^{1 / p} \mathrm{~d} \tau^{\prime}\right)^{p}}{\left(\frac{1}{\bar{\tau}} \int_{0}^{\bar{\tau}} g^{q} \mathrm{~d} \tau^{\prime}\right)^{1 / q}}\right\}^{a} .
$$

Since $p, q>1$ we can use the inequalities

$$
\begin{aligned}
& \left(\frac{1}{\bar{\tau}} \int_{0}^{\bar{\tau}} g^{1 / p} \mathrm{~d} \tau^{\prime}\right)^{p} \leq \frac{1}{\bar{\tau}} \int_{0}^{\bar{\tau}} g \mathrm{~d} \tau^{\prime}, \\
& \left(\frac{1}{\bar{\tau}} \int_{0}^{\bar{\tau}} g^{q} \mathrm{~d} \tau^{\prime}\right)^{1 / q} \geq \frac{1}{\bar{\tau}} \int_{0}^{\bar{\tau}} g \mathrm{~d} \tau^{\prime},
\end{aligned}
$$

which are particular cases of Holder's inequality. These inequalities are strict unless $g$ is a constant in which case $a(\tau)=0$ in $[0, \bar{\tau}]$. Plugging these in Eq. (26) we obtain $t(\bar{\tau})<\bar{\tau}$ unless the acceleration vanishes in the interval in which case $t(\bar{\tau})=\bar{\tau}$ and hence $C_{1}$ is at rest in $K$.

\subsection{The non-relativistic case}

In the limit $c \rightarrow+\infty$, whatever the value of $\beta \neq 0$, we have $|\beta|>1 / c$, thus we can suspect that in the non-relativistic case $t(\bar{\tau})<\bar{\tau}$. However, this 
argument does not prove that in the limit it can not be $t(\bar{\tau})=\bar{\tau}$. In order to obtain this result we have to take the $c \rightarrow+\infty$ limit of Eq. (17). As we shall see a nice formal correspondence with thermodynamics arises. is

Let $E(\tau)=\int_{0}^{\tau} a\left(\tau^{\prime}\right) \mathrm{d} \tau^{\prime}$. The non-relativistic $c \rightarrow+\infty$ limit of Eq. (17)

$$
t(\bar{\tau})=\left(\int_{0}^{\bar{\tau}} e^{-\beta E\left(\tau^{\prime}\right)} \mathrm{d} \tau^{\prime}\right) e^{\beta\left\{\frac{\int_{0}^{\bar{\tau}} e^{-\beta E\left(\tau^{\prime}\right) E\left(\tau^{\prime}\right) \mathrm{d} \tau^{\prime}}}{\int_{0}^{\bar{\tau}} e^{-\beta E\left(\tau^{\prime}\right)} \mathrm{d} \tau^{\prime}}\right\}} .
$$

This equation could have been obtained in a way analogous to that followed in the relativistic case by writing (see (12))

$$
\begin{aligned}
t(\bar{\tau}) & =\int_{0}^{\bar{\tau}} e^{-\beta v(\tau)} \mathrm{d} \tau, \\
0=x(\bar{\tau}) & =\int_{0}^{\bar{\tau}} v(\tau) e^{-\beta v(\tau)} \mathrm{d} \tau, \\
v(\tau) & =v(0)+\int_{0}^{\tau} a\left(\tau^{\prime}\right) \mathrm{d} \tau^{\prime} .
\end{aligned}
$$

Indeed, from the last two equations we get $v(0)$ that replaced into the first one gives (29). Equation (29) shows that in the classical case $(\beta=0)$ there is no differential aging. We prove here that in the non-relativistic $(c=+\infty)$ case if $\beta \neq 0$ there is always differential aging in the unusual direction, $t(\bar{\tau})<\bar{\tau}$ unless $C_{0}$ 's and $C_{1}$ 's trajectories coincide in $\tau \in[0, \bar{\tau}]$.

It is convenient to introduce the "partition function"

$$
Z(\beta, \bar{\tau})=\frac{1}{\bar{\tau}} \int_{0}^{\bar{\tau}} e^{-\beta E\left(\tau^{\prime}\right)} \mathrm{d} \tau^{\prime},
$$

the "probability density",

$$
P(\tau)=\frac{\frac{1}{\bar{\tau}} e^{-\beta E(\tau)}}{Z(\beta, \bar{\tau})}
$$

the "mean energy" function,

$$
\bar{E}(\bar{\tau})=\int_{0}^{\bar{\tau}} P\left(\tau^{\prime}\right) E\left(\tau^{\prime}\right) \mathrm{d} \tau^{\prime}=-\frac{\partial \ln Z}{\partial \beta},
$$

and the "entropy" function,

$$
S=\ln Z-\beta \frac{\partial \ln Z}{\partial \beta} .
$$

Then

$$
t(\bar{\tau})=\bar{\tau} e^{S} \quad \text { or } \quad S=\ln \frac{t(\bar{\tau})}{\bar{\tau}} .
$$

These names and the letters $E, Z, S$, follow from the striking formal analogy of these formulas with thermodynamics. The letter $\beta$ was chosen keeping in mind this analogy. We shall consider this correspondence only as a 
technical tool that will allow us to use well known results from thermodynamics. From Eq. (16) we see that in the non-relativistic limit

$$
v(0)=-\bar{E}=\frac{\partial \ln Z}{\partial \beta},
$$

which gives a kinematical interpretation for the mean energy function. If $\beta=0$ one expects to recover the classical case. Indeed we find that

$$
v(0)=-\frac{1}{\bar{\tau}} \int_{0}^{\bar{\tau}} \int_{0}^{\tau} a\left(\tau^{\prime}\right) \mathrm{d} \tau^{\prime} \mathrm{d} \tau
$$

or $\int_{0}^{\bar{\tau}} v(\tau) \mathrm{d} \tau=0$, where $v(\tau)=v(0)+\int_{0}^{\tau} a\left(\tau^{\prime}\right) \mathrm{d} \tau^{\prime}$ is the non-relativistic limit of (15). That is, the initial velocity is determined by the nonrelativistic condition that $x(\bar{T})=x(0)=0$. Now, we want to determine if differential aging occurs.

Consider the "energy variance",

$$
\overline{(\Delta E)^{2}}=\overline{(E-\bar{E})^{2}}=\overline{(E)^{2}}-\bar{E}^{2}=-\frac{\partial \bar{E}}{\partial \beta}=\frac{\partial^{2} \ln Z}{\partial \beta^{2}} .
$$

Since, by definition, the variance is positive we have

$$
\frac{\partial^{2} \ln Z}{\partial \beta^{2}} \geq 0, \quad-\frac{\partial \bar{E}}{\partial \beta} \geq 0 .
$$

We obtain for the "entropy"

$$
\frac{\partial S}{\partial \beta}=-\beta \frac{\partial^{2} \ln Z}{\partial \beta^{2}}
$$

Since its derivative, if different from zero, is negative for positive $\beta$ and positive for negative $\beta, S$ has a maximum at $\beta=0$ where $S=0$ since $t=\bar{T}$ at that value. We conclude that if $\tilde{\beta} \neq 0$ then $S(\tilde{\beta})<0$ unless $(\partial S / \partial \beta)(\beta)=0$ for all $\beta \in[0, \tilde{\beta}]$. In this last case, however, $\overline{(\Delta E)^{2}}=0$, that is $E(\tau)=\bar{E}$ is a constant and therefore there is no acceleration. This completes the proof.

\section{CONCLUSIONS}

We have studied the behavior of clocks in a $1+1$ dimensional spacetime endowed with a Finsler metric. The theory satisfies (a) the relativity principle, (b) the principle of constancy of the speed of light, and (c) the locality principle. We have not imposed a parity symmetry assumption or an equivalent isotropy assumption. The set of physical theories depends on two parameters, (i) the speed of light $c$ and (ii) the constant $\beta$ such that the coordinate transformation between inertial frames takes the form $x^{\prime \mu}=e^{\beta \theta} \Lambda_{\nu}^{\mu} x^{\nu}$ where $\theta=c \tanh ^{-1}(v / c)$ is the rapidity and $v$ is the relative 
velocity of the frames. We have shown that for a given $\beta \neq 0$ the differential aging has a sign that does not depend on the arbitrary motion of the accelerated frame. This result has been obtained by applying Holder's inequality to a generalized time dilation-acceleration formula. Moreover, we have proved that the sign changes crossing the critical values $\beta= \pm 1 / c$ for which the differential aging vanishes. At these values the transport of clocks defines a global time variable or absolute time $t+\operatorname{sgn}(\beta) x / c$. The non-relativistic limit has also been considered, showing that for $\beta \neq 0$ a formal correspondence with thermodynamics arises where $\beta$ plays the role of an inverse temperature.

These findings have been summarized in table 1 which shows that 2dimensional theories, although based on the same fundamental principles as higher dimensional theories, are richer than SR and exhibit, through the variation of $\beta$, a differential aging reversal.

The problem whether these results can be generalized to the $3+1$ dimensional theory considered by Bogoslovsky and others remains open and seems worth studying.

Acknowledgments. The author has been supported by INFN, grant $n^{\circ}$ 9503/02.

\section{REFERENCES}

[1] G. S. Asanov, Finsler geometry, relativity and gauge theories. (D. Reidel Publishing Co., Dordrecht, 1985).

[2] V. Berzi and V. Gorini, "Reciprocity principle and Lorentz transformations," J. Math. Phys., 10, 1518-1524, (1969).

[3] G. Yu. Bogoslovsky. "Lorentz symmetry violation without violation of relativistic symmetry," Physics Letters A, 350, 5-10 (2006).

[4] G. Yu. Bogoslovsky and H. F. Goenner, "On a possibility of phase transitions in the geometric structure of space-time," Physics Letters A, 244, 222-228 (1998).

[5] G. Yu. Bogoslovsky and H. F. Goenner, "Finslerian spaces possessing local relativistic symmetry," Gen. Relativ. Gravit., 31, 1565-1603 (1999).

[6] T. Budden, "A star in the Minkowskian sky: Anisotropic special relativity," Stud. Hist. Phil. Mod. Phys., 28, 325-361 (1997).

[7] C. Duval, G. Burdet, H. P. Künzle, and M. Perrin, "Bargmann structures and Newton-Cartan theory," Phys. Rev. D, 31, 1841-1853 (1985). 
[8] C. Duval, G. Gibbons, and P. Horváthy, "Celestial mechanics, conformal structures, and gravitational waves," Phys. Rev. D, 31, 1841-1853 (1985).

[9] J. H. Field, "A new kinematical derivation of the Lorentz transformation and the particle description of light," Helv. Phys. Acta, 70, 542-564 (1997).

[10] A. R. Lee and T. M. Kalotas, "Lorentz trasformations from the first postulate," Am. J. Phys., 43, 434-437 (1975).

[11] A. R. Lee and T. M. Kalotas, "Response to "Comments on 'Lorentz trasformations from the first postulate"," Am. J. Phys., 44, 10001002 (1976).

[12] H. Leutwyler and F. Stern, "Relativistic dynamics on a null plane," Ann. Phys., 112, 94-164 (1978).

[13] J. M. Lévy-Leblond, "One more derivation of the Lorentz transformation," Am. J. Phys., 44, 271-277 (1976).

[14] A. L. Macdonald, "World's fastest derivation of the Lorentz transformation," Am. J. Phys., 49, 483-483 (1981).

[15] S. Mahajan, "Comments on "Lorentz transformation from the first postulate"," Am. J. Phys., 44, 998-999 (1976).

[16] B. Mashhoon, "The hypothesis of locality in physics," Phys. Lett. A, 145, 147-153 (1990).

[17] B. Mashhoon, "Limitations of spacetimes measurements," Phys. Lett. A, 143, 176-182 (1990).

[18] E. Minguzzi, "Differential aging from acceleration: An explicit formula," Am. J. Phys., 73, 876-880 (2005).

[19] W. Rindler, Essential relativity. (Springer-Verlag, New York, 1977).

[20] H. Round, The differential geometry of Finsler spaces. (Springer, Berlin, 1959).

[21] D. A. Sardelis, "Unified derivation of the Galileo and the Lorentz transformations," Eur. J. Phys., 3, 96-99 (1982).

[22] R. Weinstock, "Derivation of the Lorentz-transformation equations without a linearity assumption," Am. J. Phys., 32, 260-264 (1964). 